\title{
Advanced method for the accurate measurement of tilt angle in a transmission electron microscopy goniometer
}

\author{
Ji-Hyun Lee, Hoyoung Suh, Sang-Gil Lee, Jin-Gyu Kim and Seung Jo Yoo (D)
}

\begin{abstract}
Background: In order to improve the reliability of the electron tomography (ET) technique, which reveals threedimensional information of nanostructured materials from a series of tilted two-dimensional images, it is essential that the mechanical tilt angle be accurately measured by the transmission electron microscopy (TEM) goniometer.

Findings: In this study, a calibration specimen was fabricated by nanohole patterning using a focused ion beam in order to determine mechanical tilt angles. The TEM goniometer tilt-angle accuracies were directly confirmed by measuring the changing areas of the projected nanosized hole. New calibration equations were developed and applied for the accurate determination of tilt angle.
\end{abstract}

Conclusion: We expect that the calibration specimen will effectively determine and correct the mechanical tilt angles in TEM goniometers leading to improvements in the ET technique.

Keywords: Electron tomography, Calibration specimen, Goniometer, Nanohole patterning, Focused ion beam

\section{Introduction}

Transmission electron microscopy (TEM) is an essential tool for the quantitative structural analysis of nanostructured materials, due to its ability to directly visualize individual nanosized objects. In particular, structural information such as specimen shape, size, and distribution can be analyzed from the directly obtained images (Mühlfeld et al. 2007). However, projected twodimensional (2D) images acquired from threedimensional (3D) nanostructured materials can provide limited information, and sometimes, information is missing; the development of nanotechnology requires the relationship between the 3D structure and properties of the material to be characterized on the nanometer scale (Ercius et al. 2015). In order to overcome the lack of information provided by 2D images, the electron tomography (ED) technique has been employed to produce reconstructed $3 \mathrm{D}$ images. In this technique, multiple $2 \mathrm{D}$ images (normally $50-150$ images), acquired by tilting the nanosized object in $1^{\circ}$ or $2^{\circ}$ intervals over a wide range

\footnotetext{
* Correspondence: sjyoo78@kbsi.re.kr

Electron Microscopy Research Center, Korea Basic Science Institute (KBSI), 169-148 Gwahak-ro, Yuseong-gu, Daejeon 34133, South Korea
}

of angles, are required in order to reconstruct a highquality 3D image. The exact alignment of a series of tilted 2D images prior to the construction of the 3D image in a manner that avoids image distortion is also important for improving 3D information. Furthermore, an accurate TEM goniometer is required for the acquisition of the tilted 2D images in order to prevent image misalignment (Hayashida et al. 2014). However, errors between the actual and TEM goniometer-displayed tilt angles may occur due to mechanical imperfections. For this reason, it is necessary to accurately measure and correct the tilt angles measured by the TEM goniometer (Hayashida et al. 2011). Although monocrystalline diffraction patterns or Kikuchi diffraction patterns have been used to measure mechanical tilt angles in a previous report (Shaw and Hills 1981), the method is not applicable to all types of TEM specimens and currently manufactured transmission electron microscopes. Therefore, an advanced method for the measurement of the actual tilt angle by a TEM goniometer is required. In this study, we report the development of a new method for the direct and accurate determination of tilt angles by a TEM goniometer. 


\section{Experimental}

\section{Fabrication of nanohole patterns}

A nanohole with a diameter of $500 \mathrm{~nm}$ was fabricated on a SiN membrane TEM window grid (SN100-A50Q10, SiMPore Inc.) using a focused ion beam (FIB) (Quanta $3 \mathrm{D}$ FEG, FEI) at an accelerating voltage of $30 \mathrm{kV}$, ion current of $10 \mathrm{pA}$, and dwell time of $15 \mu \mathrm{s}$. Prior to nanohole patterning, the SiN membrane was coated with a several-nm-thick Au layer using a sputter coater (SCD 055, BAL-TEC) to reduce the effect of charging caused by the insulating $\mathrm{SiN}$ film.

\section{Nanohole pattern imaging}

The nanosized hole was analyzed by Cs-corrected TEM (Libra 200 HT Mc, Carl Zeiss). A series of tilted 2D images of the nanosized hole was obtained over the $-50^{\circ}$ to $+50^{\circ}$ range in $2^{\circ}$ increments. In each $2 \mathrm{D}$ image, the projected area of the nanosized hole was measured using the particle analysis tool available in the DigitalMicrograph (DM) software package (Gatan Inc.).

\section{Results and discussion}

\section{Establishment of the calibration equation}

Tilt angle can simply be determined from the projected area of the hole using the following equation:

$$
\theta=\cos ^{-1} \frac{A_{T}}{A_{I}}
$$

where $\theta$ is the tilt angle, $A_{T}$ is the projected area of the nanohole tilted at angle $\theta$, and $A_{I}$ is the initial area of the nanohole. However, this equation is only applicable when the nanohole is very thin. Indeed, the nanohole pattern that we used was $50 \mathrm{~nm}$ thick (the thickness of the SiN membrane). Therefore, we modified the formula to take into account the thickness of the nanohole through simple geometrical calculations. A schematic diagram of the developed protocol is presented in Fig. 1. When the incident beam is perpendicular to plane $\mathrm{A}$, the tilt angle between the nanohole and plane $\mathrm{A}$ is $\theta$. The area of the nanohole projected by the transmitted beam $\left(A_{T}\right)$ is equal to the overlap area between the red and blue circles multiplied by $\cos \theta$, as shown in Fig. 1b, where the overlap area, $A_{O}$, is defined as

$$
A_{O}=2 \times\left(A_{C}-A_{I T}\right)=r^{2}(2 \alpha-\sin 2 \alpha)
$$

and $A_{T}$ and $\theta$ are derived as shown below:

$$
\begin{aligned}
& A_{T}=A_{O} \cos \theta=r^{2}(2 \alpha-\sin 2 \alpha) \cos \theta \\
& \theta=\cos ^{-1} \frac{A_{T}}{r^{2}(2 \alpha-\sin 2 \alpha)}=\cos ^{-1}\left[\frac{A_{T}}{A_{I}} \frac{\pi}{(2 \alpha-\sin 2 \alpha)}\right]
\end{aligned}
$$

In Eq. (2), $A_{C}$ is the area of the yellow sector, $A_{I T}$ and $\alpha$ are the area and vertex angle of the green isosceles triangle, respectively, and $r$ is the radius of the hole. Angle $\alpha$ is obtained by introducing the additional variables displayed in Fig. 1a as follows:

$$
\begin{aligned}
& x=\frac{t \tan \theta}{2} \\
& \alpha=\cos ^{-1} \frac{t \tan \theta}{2 r}
\end{aligned}
$$

where $t$ is the thickness of the nanohole.

If the nanohole is very thin $(r>>t)$, then Eq. (4) collapses to Eq. (1) as expected. From Eqs. (4) and (5), we see that $\theta$ is a function of $A_{O}, t$, and $r$. Since $t$ and $r$ are known variables determined from the features of the nanohole, we only need to consider the projected area of the tilted nanohole in order to calculate the tilt angle.

To verify the reliability of this equation, tilt angles were calculated using a simulated 3D model with an aspect ratio of 0.1 between diameter and thickness. Figure $2 \mathrm{a}$ shows the representative $3 \mathrm{D}$ model images
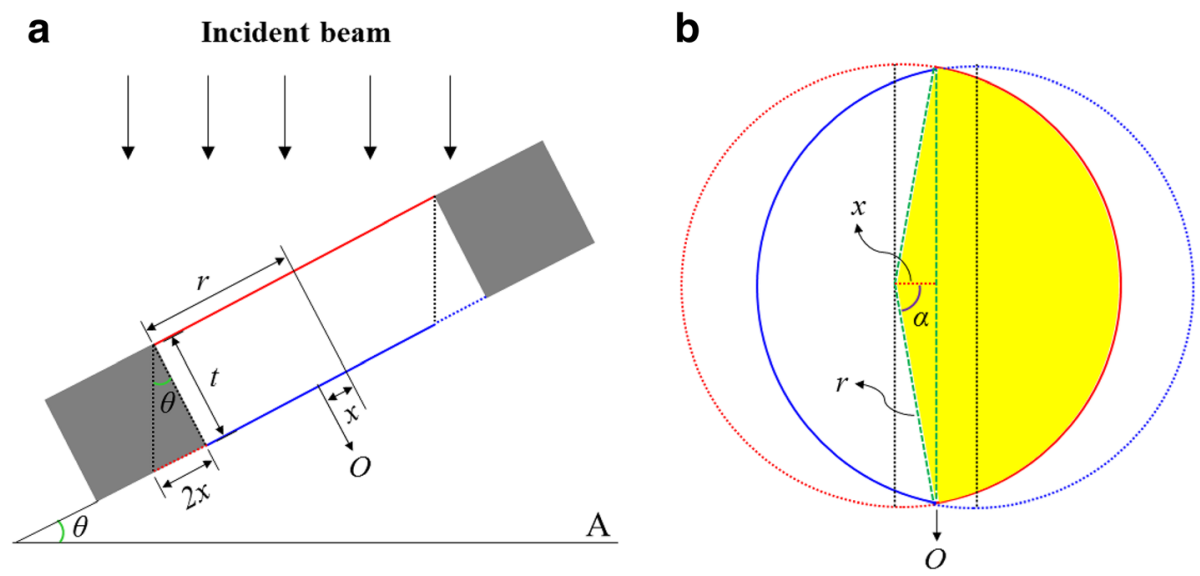

Fig. 1 a Schematic diagram of the tilted hole. $\mathbf{b}$ The projected object for the calculation of the projection area 

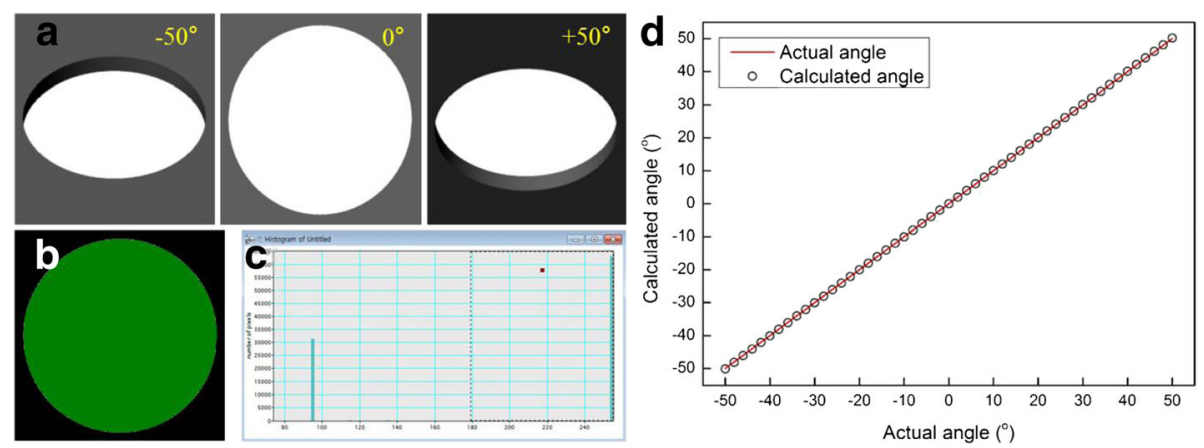

Fig. 2 a 3D-simulated images at different tilt angles. b Selected hole area. c Corresponding histogram. d Calculated vs. actual angles

obtained at different tilt angles in which the models were tilted in $2^{\circ}$ increments over the $-50^{\circ}$ to $+50^{\circ}$ range. The projected area of the nanohole was measured by counting the number pixels above the threshold intensity of the brightest region using the DM software, as shown in Fig. 2b, c. Finally, the numerical tilt-angle solution was determined from the acquired projected area by computer calculation. Figure $2 \mathrm{~d}$ displays the calculated tilt angle as a function of actual tilt angle. All calculated angles corresponded to the actual angle to within an error $(2 \sigma)$ of $0.6 \%$; the $R^{2}$ value of the linear fit was unity, which means that a well-defined nanohole device will lead to the correct goniometer tilt angle from the projected area fraction without the need for any external device for measuring tilt angle.

\section{Fabrication of the nanohole pattern}

To fabricate a well-defined nanohole, we used the FIB patterning technique. Figure 3a shows an SEM image that displays the morphology of the entire SiN membrane of the TEM window grid. The dark line in the blue square is an array of 200-nm-diameter holes (inset, Fig. 3a) that was formed by hair masking the Au coating to form guidelines for the location of the nanohole pattern in the center of the window (red square). For tilt-angle measurements, we fabricated a 500-nm-diameter nanohole in a 50-nm-thick $\mathrm{SiN}$ membrane, as shown in Fig. 3b.

\section{Analysis of the fabricated nanosized hole by TEM}

During the TEM analysis of the nanohole, both brightfield TEM (BF-TEM) images in conventional TEM mode were acquired, as shown in Fig. 4a, b, as well as highangle annular dark field images in scanning TEM mode (HAADF-STEM), as shown in Fig. 4d, e, in order to compare the measurement accuracies. Figure $4 \mathrm{c}, \mathrm{f}$ displays the BF-TEM and HAADF-STEM image histograms, respectively, which reveal that the HAADFSTEM images display more distinct edge contrast between the nanohole and the amorphous thin film and, consequently, the hole area was more accurately selected for measurement. Based on the analyzed results, the measurement error is significantly reduced in HAADFSTEM mode.
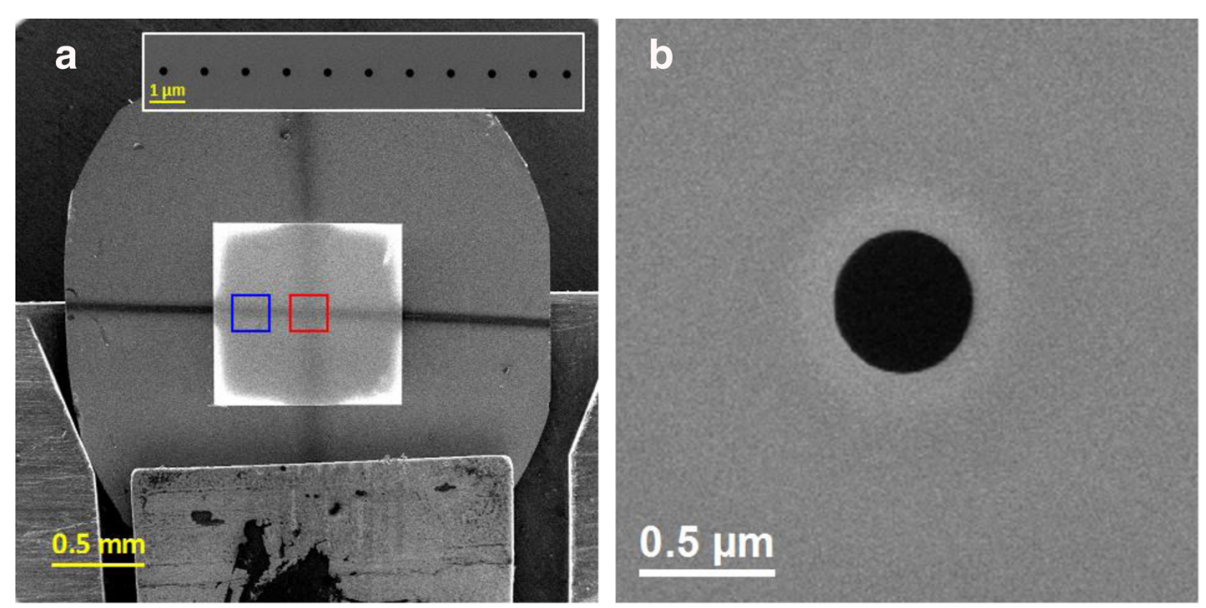

Fig. 3 a SEM image of the SiN membrane TEM window grid (the inset shows the guide-hole array). b An individual nanohole fabricated by FIB patterning 

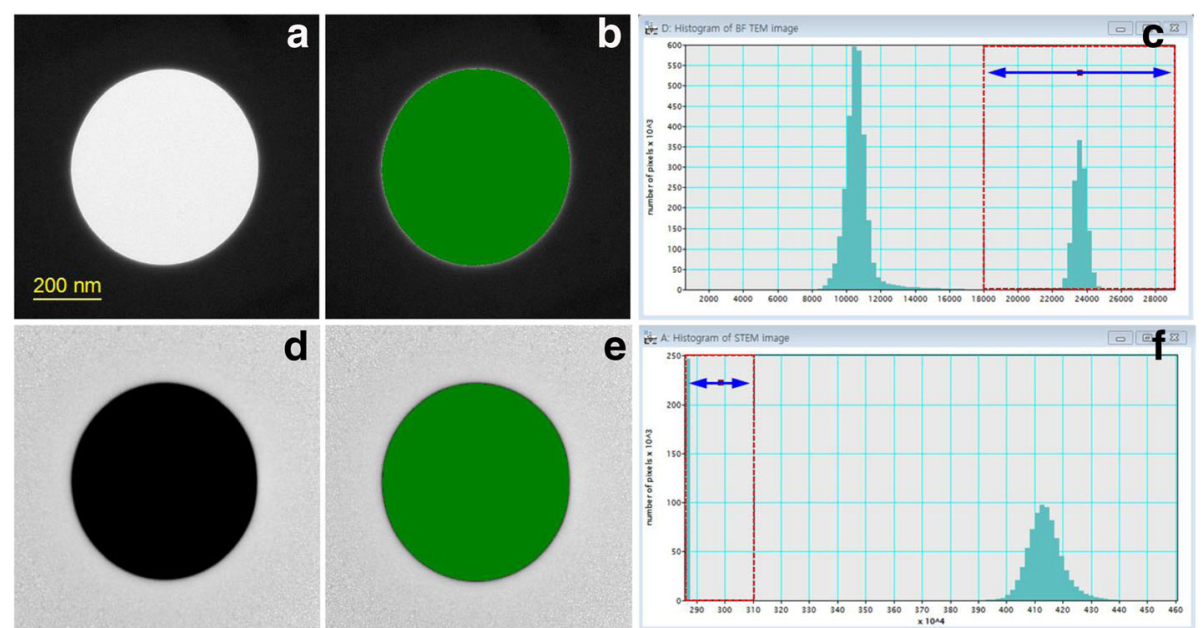

Fig. 4 The determination of projected area. a The BF-TEM image. b The selected hole area. c The corresponding TEM histogram. $\mathbf{d}$ The HAADFSTEM image. e The selected hole area. $\mathbf{f}$ The corresponding STEM histogram. $\mathbf{b}-\mathbf{c}$ The selected hole area was highlighted in green color

The fabricated nanosized hole was applied in a practical setting for the actual measurement of tilt angles by the TEM goniometer. The 500-nm nanosized hole was used to acquire a series of tilted $2 \mathrm{D}$ images. The projected area of the nanosized hole was determined in each $2 \mathrm{D}$ image, and the changes in tilt angle were calculated by computer using the equations discussed in the previous section. Figure 5 displays the calculated angle as a function of the nominal angle displayed by the goniometer. The average differences between the two angles in the 0 to $+50^{\circ}$ and 0 to $-50^{\circ}$ ranges were $1.09^{\circ}$ and $2.18^{\circ}$, respectively. The errors determined by computer calculation appear to be quite high when compared to the value of $\pm 0.02^{\circ}$ provided by the TEM manufacturer. However, since we formulated Eq. (4) assuming an ideal nanohole geometry, this observed difference is possibly due to the gentle slope of the nanohole wall.

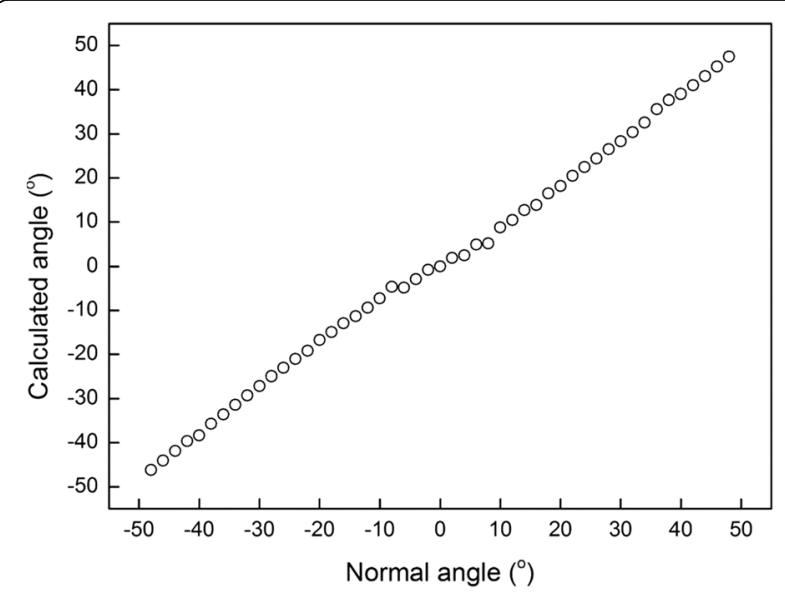

Fig. 5 Calculated angle as a function of nominal angle displayed by the TEM goniometer

\section{Conclusions}

A 500-nm-diameter nanosized hole was fabricated in a SiN membrane TEM window grid by FIB patterning. The ion beam milling conditions were optimized in order to produce the desired shape and size of the nanosized hole. In this technical note, we address a newly developed method for the measurement of the tilt angle in a TEM goniometer using a fabricated nanohole, and a simple equation that considers the nanohole geometry is introduced to accurately calculate tilt angles in a TEM goniometer. By applying this advanced method, the artificial nanohole can be as a calibration specimen to correct the mechanical tilt angles provided by a TEM goniometer. We expect that this method will increase the reliability of the ET technique for the structural analysis of nanosized objects without the need for expensive devices, although further development of the FIB technique is needed in order to improve sharp-edge control, to reduce the influence of film thickness, and to improve angular-measurement accuracies.

\section{Abbreviations}

BF: Bright field; ET: Electron tomography; FIB: Focused ion beam; HAAFD: High-angle annular dark field; STEM: Scanning transmission electron microscopy; TEM: Transmission electron microscopy

\section{Acknowledgements}

This work was supported by a Korea Basic Science Institute grant (D38612).

\section{Authors' contributions}

JGK designed and coordinated the study. JHL and SGL carried out the experiments and image processing. $\mathrm{HS}$ and SJY refined the data and drafted the manuscript. All authors have read and approved the final manuscript. 


\section{Publisher's Note}

Springer Nature remains neutral with regard to jurisdictional claims in published maps and institutional affiliations.

Received: 13 November 2017 Accepted: 22 January 2018

Published online: 29 January 2018

\section{References}

Ercius P, Alaidi O, Rames MJ, Ren G. Electron tomography: a three-dimensional analytic tool for hard and soft materials research. Adv Mater. 2015;27:5638-63.

Hayashida M, Malac M, Bergen M, Egerton RF, Li P. Accurate measurement of relative tilt and azimuth angles in electron tomography: a comparison of fiducial marker method with electron diffraction. Rev Sci Instrum. 2014;85:083704.

Hayashida M, Terauchi S, Fujimoto T. Calibration method of tilt and azimuth angles for alignment of TEM tomographic tilt series. Rev Sci Instrum. 2011:82:103706.

Mühlfeld C, Rothen-Rutishauser B, Vanhecke D, Blank F, Gehr P, Ochs M.

Visualization and quantitative analysis of nanoparticles in the respiratory tract by transmission electron microscopy. Part Fibre Toxicol. 2007:4:11.

Shaw PJ, Hills GJ. Tilted specimen in the electron microscope: a simple specimen holder and the calculation of tilt angles for crystalline specimens. Micron. 1981;12:279-82

\section{Submit your manuscript to a SpringerOpen ${ }^{\circ}$ journal and benefit from:}

- Convenient online submission

- Rigorous peer review

- Open access: articles freely available online

- High visibility within the field

Retaining the copyright to your article

Submit your next manuscript at $>$ springeropen.com 